\title{
Risk of Fall in Patients during the Early Months after Total Knee Arthroplasty
}

\author{
Yoshinori Hiyama ${ }^{1,2 *}$ and Shuichi Okada ${ }^{2}$
}

${ }^{1}$ Anshin Hospital, Minatojima, Minamimachi, Kobe, Hyogo, Japan

${ }^{2}$ Graduate school of Human Development and Environment, Kobe University, Japan

\begin{abstract}
Objective: Falls are among the most common causes of injury in elderly people, whereas little is known about the risk of falls during the early months after hospital discharge in people undergoing Total Knee Arthroplasty (TKA) Therefore, this study aimed to assess risk of falling in patients during the early months after TKA.

Methods: Seventy patients undergoing primary TKA for knee osteoarthritis participated in this study. The risk of falling as assessed using knee Range Of Motion (ROM), the knee extension strength, 10-m walking test, Timed Up \& Go (TUG) test, and Sit-to-Stand test findings and falls efficacy using the modified Falls Efficacy Scale (mFES) were evaluated 1 month before surgery, on the day of hospital discharge ( 5 days after surgery), and 1, 2, and 3 months after surgery.

Results: Of the 70 patients, $3(4.3 \%)$ reported falls in the first 3 months after surgery. Patients who underwent TKA experienced an expected worsening of knee ROM, knee extension strength, and performance on the 10-m walking, TUG, and Sit-to-Stand tests on the day of discharge $(p<0.001)$. All measures of physical function were comparable to the preoperative levels 3 months after surgery. The mFES scores displayed no significant differences among the assessments performed before surgery, on the day of discharge, and 1,2, and 3 months after surgery.

Conclusion: Despite worsening of physical function after TKA, patients displayed similar falls efficacy to that observed preoperatively. These results indicated that patients undergoing TKA might underestimate their risk of falls during the early months after hospital discharge despite the likelihood of an increased risk of falls because of declines in physical function including strength, gait ability, and mobility. The clinical implication of this finding is that falls prevention interventions are needed prior to hospital discharge.
\end{abstract}

Keywords: Falls; Falls risk; Knee osteoarthritis; Total knee arthroplasty; Falls efficacy

\section{Introduction}

Total Knee Arthroplasty (TKA) is the most common surgical intervention for end-stage knee Osteoarthritis (OA), and it offers patients pain relief, functional recovery, and improved quality of life [1,2]. An estimated 70,000 TKA procedures are performed yearly in Japan, and the number increases annually. Early initiation of rehabilitation therapy after TKA, such as interventions to increase joint Range of Motion (ROM) and quadriceps strength, is becoming popular, and it is considered important for obtaining the maximum benefit from knee arthroplasty [3-5]. Moreover, the early initiation of rehabilitation is beneficial for early hospital discharge [3-5]. Early hospital discharge is expected to lower the risk of hospital-acquired infection and allow patients to quickly return to their daily life activities after surgery [6].

Falls are among the most common causes of injury in elderly people, and knee OA is an important risk factor for falls, with more than $50 \%$ of people with knee OA reporting falls in the past year [7-11]. Despite the high prevalence of falls in people with knee OA, little is known about the risk of falls in people following TKA. Levinger et al. assessed the risk of falls in people with knee OA before surgery and at 4 months following surgery and concluded that people undergoing TKA were at increased risk of falls both prior to and 4 months following surgery [12]. Moreover, Matsumoto et al. found that people undergoing TKA were more likely to fall than healthy elderly people 1 year after surgery [13]. However, the risk of falls in people who underwent TKA during the early months after hospital discharge is unclear. Recently, there has been a trend toward early hospital discharge; therefore, it is important to assess the risk of falls during the early months after hospital discharge. Thus, the purpose of this study was to assess the risk of falls in patients with knee OA before and at 1,2 , and 3 months after TKA.

\section{Methods}

The target population consisted of patients undergoing primary TKA for knee OA (Table 1); in total, 70 patients (21 men and 49 women) who underwent primary TKA for knee OA were recruited and tested prior to the surgery, on the day of discharge, and at 1, 2, and 3 months following surgery. The inclusion criteria were as follows: (i) completion of primary unilateral TKA for knee OA staged as grade 3 or 4 based on the Kellgren and Lawrence radiographic grading system (radiographic evaluation was performed by an independent experienced assessor) [14]; (ii) no demonstrable symptoms in the hip, ankle, and contralateral knee while walking; (iii) ability to walk independently; and (iv) provision of informed consent to participate

*Corresponding author: Yoshinori Hiyama, Anshin Hospital, 1-4-12, Minatojima Minamimachi, Kobe, Hyogo, 650-0047, Japan Tel: +81-78-304-5252; Fax: +81-78304-5222; E-mail: yoshinori.hiyama@gmail.com

Received August 19, 2014; Accepted October 03, 2014; Published October 13 2014

Citation: Hiyama Y, Okada S (2014) Risk of Fall in Patients during the Early Months after Total Knee Arthroplasty. J Arthritis 3: 139. doi:10.4172/2167-7921.1000139

Copyright: $\odot 2014$ Hiyama Y, et al. This is an open-access article distributed under the terms of the Creative Commons Attribution License, which permits unrestricted use, distribution, and reproduction in any medium, provided the original author and source are credited. 


\begin{tabular}{|l|c|}
\hline Characteristics & Participants $\mathbf{( n = 7 0 )}$ \\
\hline Age (years) & $73.5 \pm 5.2$ \\
\hline range & $64-78$ \\
\hline Women, $\mathrm{n}(\%)$ & $62(88.6)$ \\
\hline Height $(\mathrm{cm})$ & $154.5 \pm 10.2$ \\
\hline Weight $(\mathrm{kg})$ & $61.3 \pm 10.5$ \\
\hline Body mass index $\left(\mathrm{kg} / \mathrm{m}^{2}\right)$ & $25.0 \pm 6.8$ \\
\hline Grade of osteoarthritis in the involved limb, $\mathrm{n}(\%)$ & \\
\hline Grade 3 & $10(14.3)$ \\
\hline Grade 4 & $60(85.7)$ \\
\hline
\end{tabular}

Data are presented as the mean \pm SD.

Table 1: Characteristics of the study participants.

in this study. The exclusion criteria were as follows: (i) musculoskeletal involvement other than unilateral TKA limiting patient function; (ii) diagnosis of uncontrolled blood pressure, diabetes mellitus, or neurological disorders such as Parkinson disease; (iii) chronic disorders such as chronic dizziness, cardiovascular disorders, and low blood pressure; (iv) anatomical abnormality such as patella alta; (v) body mass index $>40$ (morbidly obese); and (vi) planning to undergo surgery on the uninvolved knee. This was a prospective cohort study. The Institutional Review Board approved all procedures performed in this study before testing, and all patients provided written informed consent before participating.

\section{Procedures}

Regular inpatient physical therapy was initiated on postoperative day 1 , followed by a standard protocol of ambulation exercises and activities of daily living exercises. In the morning on postoperative day 1 , patients initially performed ambulation exercise using a walker and full weight bearing was allowed as tolerated. After achieving independent ambulation with a walker, ambulation exercise using a cane was introduced in the morning on postoperative day 2. On postoperative day 3 , patients achieved independent ambulation with a cane, and patients were permitted to practice descending and ascending stairs in the morning on postoperative day 4 . The mobility-related goal at discharge was independent and safe ambulation and the ability to ascend and descend stairs while holding the railing. Physical therapists and nurses defined independent ambulation if patients could walk $45 \mathrm{~m}$ with assistance [15].

\section{Falls risk assessment}

The number of falls experienced by the participants in the 3 months prior to and after surgery was assessed. A fall was defined as an unexpected event where a person falls to the ground from an upper level or the same level [16]. Falls resulting from extraordinary environmental factors (e.g., traffic accidents or falls while riding a bicycle) were excluded.

Knee extension strength, the $10-\mathrm{m}$ walking test, the Timed Up and Go (TUG) test, and the Sit-to-Stand test were used to assess the risk of falls [17-19]. To ensure the reliability of outcomes and prevent measurement error, all assessments were performed by a single physical therapist.

Knee extension strength was measured as the peak isometric knee extension torque $(\mathrm{Nm} / \mathrm{kg})$ using a hand-held dynamometer ( $\mu$ Tas F1; ANIMA, Chofu, Japan). Participants were seated, with their hands on their laps, on a chair designed to stabilize the body and minimize synkinetic movements. The knee joint was held at an angle of $75^{\circ} \mathrm{C}$, and a belt was used to restrain the dynamometer to enhance the reliability of isometric leg muscle strength measurements [20]. Participants were asked to extend their knees (by using the command "kick as hard as possible") for $3 \mathrm{~s}$ [21]. During each test, consistent verbal encouragement was provided to the subjects. The peak torque was estimated as the product of force and the distance between the attachment of the dynamometer (at the lateral malleolus) and the center of rotation of the knee joint. Two attempts of maximal contraction were performed, and the greater of the 2 measurements was recorded and normalized to body weight. The quadriceps strength of the surgical limb was measured. The intraclass correlation coefficient for intratester measurement reliability was 0.97 .

For the 10-m walking test, participants were instructed to walk at a self-selected, comfortable pace along a 16-m long, 2-m wide hallway [17]. Gait time was measured to one-hundredth of a second using a stopwatch as the participant walked in the central $10-\mathrm{m}$ portion of the walkway, which was identified for the examiner using tape markings. The time was recorded for 2 trials and averaged as the $10-\mathrm{m}$ walking time. The validity and reliability of the $10-\mathrm{m}$ walking test have been reported [22]. For the TUG test, participants were instructed to stand up from a chair with no seat arms and a seat height of $40 \mathrm{~cm}$, walk a distance of $3 \mathrm{~m}$ at a normal pace, turn, walk back to the chair, and return to a seated position [18]. The time was recorded to one-hundredth of a second using a stopwatch, and time to complete the TUG test of 2 trials were averaged to obtain the TUG score. This test is reliable and valid for assessing group changes among inpatients undergoing orthopedic rehabilitation [23]. For the Sit-to-Stand test, participants began by sitting with their back against a chair with no seat arms and a seat height of $40 \mathrm{~cm}$. They were asked to stand up from the chair once. If successful, they were instructed to stand up and sit down 5 times as quickly as possible. Timing began when the examiner said, "Go" and stopped when the participant's buttocks touched the seat on the fifth repetition [19]. They were instructed not to touch the seat during each repetition. The Sit-to-Stand Test was timed in seconds only once for fear of pain.

Assessment of ROM followed procedural guidelines recommended by MacDougall et al. for the determination of start and end positions [24]. Passive knee flexion and extension ROM were evaluated in the supine position and knee extension ROM was assessed with the patient's heel propped off the treatment table on a block. The end of passive ROM was determined once the tester felt resistance or the subject vocalized discomfort. Angular displacement was measured using a standard long-arm goniometer. The lateral epicondyle and the greater trochanter of the femur, and the lateral malleolus of the ankle were identified and the goniometer then positioned. The passive movement was performed and the goniometer was then replaced in the same position, and the measurement was recorded. The same tester performed all measurements. The intraclass correlation coefficients for intratester measurement reliability were 0.96 and 0.94 for flexion and extension, respectively. These results agreed with those of previous studies [25].

\section{Falls efficacy}

The modified Falls Efficacy Scale (mFES) is a self-reported measure of an individual's self-perceived ability to complete a task without falling [26]. The mFES consists of 14 items aimed at assessing falls efficacy during both indoor and outdoor activities. Examples of the 10 items assessing indoor activities are getting dressed and bathing; conversely, crossing roads and using public transport are examples of the 4 outdoor activities assessed. The total score on the mFES can range from 10 to 40 , with low scores indicating greater confidence. The 
mFES has been used in empirical research investigating falls within a community-dwelling elderly population [27].

\section{Statistical analysis}

Pre-protocol analysis was conducted after excluding patients who withdrew from the study. Prior to data analysis, all variables were assessed for normality using the Kolmogorov-Smirnov test. All variables followed the normal distribution; therefore, analysis of variance with the Tukey-Kramer post hoc test was used to assess the differences in mFES, knee ROM, knee extension strength, and 10-m walking test, TUG test, and Sit-to-Stand test findings between before and 1, 2 and 3 months after surgery.

Subsequently, we calculated the percent change in mFES, knee ROM, knee extension strength, and 10-m walking test, TUG test, and Sit-to-Stand test findings. Percent change was calculated by subtracting the preoperative value from the postoperative value at each testing time and dividing that difference by the preoperative value [21]. As faster times on the 10-m walking test, TUG test, and Sit-to-Stand test represent better performance, we altered the determination of percent change for the test so that a positive value for percent change represented improvement for all measures [21]. Therefore, the percent changes in the 10-m walking, TUG, and Sit-to-Stand test times were calculated by subtracting the postoperative value at each testing time from the preoperative value and dividing that difference by the preoperative value. All statistical analyses were performed using SPSS Version 22.0 (IBM, Tokyo, Japan), and a $P$ value less than 0.05 was considered statistically significant.

\section{Results}

All 70 patients completed the assessment preoperatively and at 1,2, and 3 months after surgery. Of these patients, no patients reported falls in the 3 months prior to surgery, whereas 3 patients (4.3\%) reported falls in the 3 months after surgery.

Knee ROM (flexion: $\mathrm{F}=294.08, d f=4,345, p<0.001$; extension: $\mathrm{F}=33.68, d f=4,345, p<0.001)$, knee extension strength $(\mathrm{F}=88.33, d f=4$, $345, p<0.001)$, and the 10 - $\mathrm{m}$ walking test $(\mathrm{F}=32.57, d f=4,345, p<0.001)$, TUG test $(\mathrm{F}=84.67, d f=4,345, p<0.001)$, and Sit-to-Stand test times $(\mathrm{F}=47.26, d f=4,345, p<0.001)$ demonstrated a main effect for change over time (Table 2).

Compared to the preoperative value, knee flexion ROM was $28 \%$ lower in the participants on the day of discharge. Improvements in knee flexion ROM were observed at each subsequent assessment compared with the findings on the day of discharge $(p<0.001)$. Knee flexion ROM was decreased by $5.6 \%$ from the preoperative value at the final test; however, there was no significant difference between the preoperative and 3-month assessment values ( $p=0.95)$. Knee extension ROM in the assessment on the day of discharge was 6 degrees better than that before surgery $(p<0.001)$. There were no significant differences observed at each subsequent assessment.

Knee extension strength was $58 \%$ lower than the preoperative value in the test performed on the day of discharge $(p<0.001)$ (Figure 1). Improvements in knee extension strength were noted in each subsequent assessment, and there was no significant difference in knee extension strength between the preoperative and 3-month postoperative assessments $(p=0.92)$.

The 10-m walking, TUG, and Sit-to-Stand test times were significantly longer in the assessment performed on the day of discharge than in the preoperative tests $(p<0.001)$ (Figure 1). Performance on these tests improved significantly with each subsequent testing period up to the 3 -month test $(p<0.001)$. Preoperative testing times did not differ significantly from the times of the last testing session for the 10 -m walking ( $p=0.97)$, TUG $(p=0.09)$, and Sit-to-Stand tests $(p=0.11)$.

No significant differences in mFES scores were observed among the before surgery, day of discharge, and 1-, 2-, and 3-month assessments $(\mathrm{F}=2.07, d f=4,345, p=0.08$ ) (Figure 1).

\section{Discussion}

As there is a trend toward early hospital discharge, previous studies investigated the risk of falling at 4 months or 1 year after TKA, but the risk of falling during the early months after hospital discharge remains unclear $[12,13]$. We therefore assessed the risk of falling during the early months after hospital discharge in patients who underwent TKA.

The results of this study demonstrated patients who underwent TKA experienced a worsening of knee flexion ROM, knee extension strength, and performance on the 10-m walking, TUG, and Sit-to-Stand tests after surgery, which is consistent with the results of a previous study illustrating that patients who undergo TKA experience a decline in physical function [21]. Although knee extension ROM on the day of discharge improved better than that before surgery, change of ROM was only 6 degrees and had no clinical effect. Previous studies reported that knee extension strength, difficulty rising from a chair, reduced walking speed, and decreased mobility, are strongly associated with the risk of falls [28-32]. Therefore, the results of this study indicated that patients undergoing TKA were at greater risk of falls during the early months after hospital discharge than before surgery.

On the contrary, mFES scores were not significantly different between before and after surgery. The mFES represents an individual's self-perceived ability to complete a task without falling [26], and patients undergoing TKA appear to maintain their falls efficacy at a

\begin{tabular}{|c|c|c|c|c|c|}
\hline \multirow{2}{*}{ Physical function } & \multirow{2}{*}{ Preoperative } & \multicolumn{4}{|c|}{ Postoperative } \\
\hline & & The day of discharge & 1 month & 2 months & 3 months \\
\hline \multicolumn{6}{|l|}{ Range of motion } \\
\hline Flexion (degrees) & $127 \pm 12$ & $91 \pm 14$ & $108 \pm 11$ & $115 \pm 9$ & $119 \pm 7$ \\
\hline Extension (degrees) & $-8 \pm 7$ & $-2 \pm 5$ & $-2 \pm 4$ & $-2 \pm 3$ & $-2 \pm 2$ \\
\hline Knee extension strength $(\mathrm{Nm} / \mathrm{kg})$ & $0.91 \pm 0.27$ & $0.37 \pm 0.18$ & $0.65 \pm 0.22$ & $0.81 \pm 0.23$ & $0.94 \pm 0.21$ \\
\hline 10-m walking test (s) & $9.7 \pm 1.6$ & $14.6 \pm 5.5$ & $11.8 \pm 3.2$ & $10.5 \pm 2.0$ & $9.6 \pm 1.5$ \\
\hline Timed Up and Go Test (s) & $13.7 \pm 2.6$ & $20.0 \pm 3.7$ & $15.0 \pm 2.8$ & $13.6 \pm 2.3$ & $12.6 \pm 2.2$ \\
\hline The Sit-to-Stand test (s) & $15.3 \pm 3.6$ & $23.5 \pm 9.0$ & $16.4 \pm 4.0$ & $14.6 \pm 3.0$ & $13.3 \pm 2.8$ \\
\hline mFES score & $18.8 \pm 3.5$ & $18.7 \pm 4.4$ & $18.5 \pm 4.0$ & $17.4 \pm 3.4$ & $16.1 \pm 3.5$ \\
\hline
\end{tabular}

Data are presented as the mean $\pm S D$.

mFES: modified Falls Efficacy Scale 


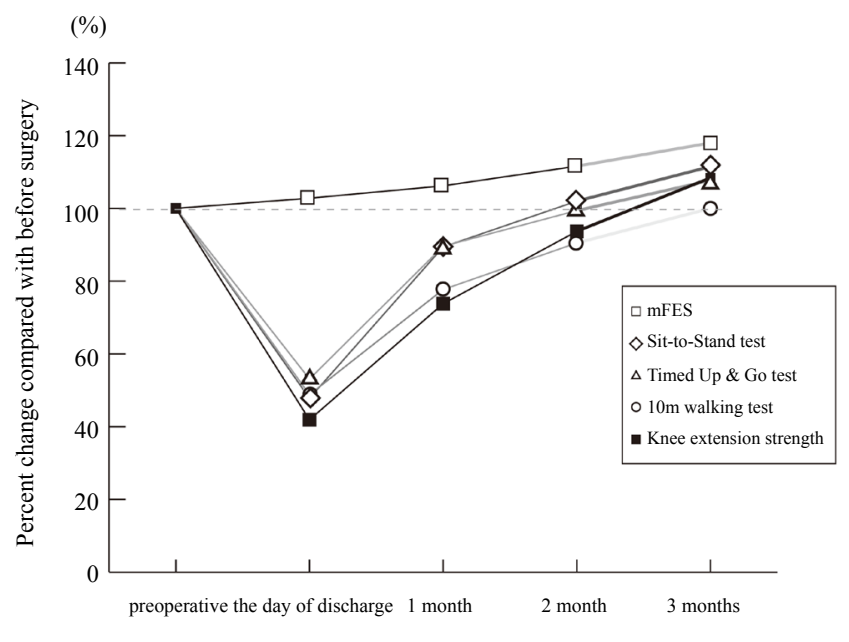

Testing Time

Figure 1: A comparison of the modified Falls Efficacy Scale (mFES) and physical function assessments including knee extension strength, 10-m walking test, Timed Up \& Go test, and Sit-to-Stand test assessments performed before and after surgery.

level comparable to that before surgery despite experiencing a decline in physical function. Therefore, patients undergoing TKA may underestimate their risk of falls during the early months after hospital discharge despite have an increased risk of falls because of a decline in physical function. Fortinsky et al. found that nearly half of older adults with a history of fall-related incidents have higher balance confidence despite having a high fall risk based on fall history [33]. Moreover, Robinovitch et al. reported that falls in older persons who overestimated their physical ability might partially be because of a lack of awareness about declines of physical ability [34]. In this study, patients after TKA had greater confidence to complete a task without falling despite a high risk of falling because of declines in physical function during the early months after hospital discharge. Therefore, such overconfidence may further increase the risk of falling in patients after TKA during the early months.

This study has several limitations. The first limitation is the lack of a physical assessment of balance. Reduced dynamic balance is strongly associated with the risk for falls, and patients may have a problem of balance ability during the early months after TKA [28,29,32,35]. Second, we did not have longitudinal data to determine whether falls occurred for a long period. Further research is needed to investigate the relationship between falls efficacy and physical function in the long term. The third limitation is the accuracy of assessment of knee extension strength and knee ROM. Although we showed high ICC in the both tests, the tests by using hand-held dynamometer and goniometer may potentially lack the accuracy. Despite these limitations, the finding in the current study indicates that patients undergoing TKA have an increased risk of falls because of overconfidence during the early months after hospital discharge. Physical therapists should call patient's attention to falls and rise patient's awareness of the risk of falls compatible with their physical function during early months after discharge.

\section{References}

1. Callahan CM, Drake BG, Heck DA, Dittus RS (1994) Patient outcomes following tricompartmental total knee replacement. A meta-analysis. JAMA 271: 13491357.
2. Kauppila $A M$, Kyllönen $E$, Ohtonen $P$, Hämäläinen $M$, Mikkonen $P$, et al (2010) Multidisciplinary rehabilitation after primary total knee arthroplasty: a randomized controlled study of its effects on functional capacity and quality of life. Clin Rehabil 24: 398-411.

3. Munin MC, Rudy TE, Glynn NW, Crossett LS, Rubash HE (1998) Early inpatient rehabilitation after elective hip and knee arthroplasty. JAMA 279: 847-852.

4. Lombardi AV Jr, Viacava AJ, Berend KR (2006) Rapid recovery protocols and minimally invasive surgery help achieve high knee flexion. Clin Orthop Relat Res 452: 117-122.

5. Labraca NS, Castro-Sánchez AM, Matarán-Peñarrocha GA, Arroyo-Morales M, Sánchez-Joya Mdel M, et al. (2011) Benefits of starting rehabilitation within 24 hours of primary total knee arthroplasty: randomized clinical trial. Clin Rehabil 25: 557-566.

6. Reilly KA, Beard DJ, Barker KL, Dodd CA, Price AJ, et al. (2005) Efficacy of an accelerated recovery protocol for Oxford unicompartmental knee arthroplasty-a randomised controlled trial. Knee 12: 351-357.

7. Blake AJ, Morgan K, Bendall MJ, Dallosso H, Ebrahim SB, et al. (1988) Falls by elderly people at home: prevalence and associated factors. Age Ageing 17 365-372.

8. Leveille SG, Bean J, Bandeen-Roche K, Jones R, Hochberg M, et al. (2002) Musculoskeletal pain and risk for falls in older disabled women living in the community. J Am Geriatr Soc 50: 671-678.

9. Sturnieks DL, Tiedemann A, Chapman K, Munro B, Murray SM, et al. (2004) Physiological risk factors for falls in older people with lower limb arthritis. J Rheumatol 31: 2272-2279.

10. Brand C, Juan AW, Lowe A, Morton C (2005) Prevalence, outcome and risk fo falling in 155 ambulatory patients with rheumatic disease. APLAR Journal of Rheumatology 8: 99-105.

11. Williams SB, Brand CA, Hill KD, Hunt SB, Moran H (2010) Feasibility and outcomes of a home-based exercise program on improving balance and gait stability in women with lower-limb osteoarthritis or rheumatoid arthritis: a pilot study. Arch Phys Med Rehabil 91: 106-114.

12. Levinger P, Menz HB, Wee E, Feller JA, Bartlett JR, et al. (2011) Physiological risk factors for falls in people with knee osteoarthritis before and early after knee replacement surgery. Knee Surg Sports Traumatol Arthrosc 19: 1082 1089.

13. Matsumoto H, Okuno M, Nakamura T, Yamamoto K, Hagino H (2012) Fall incidence and risk factors in patients after total knee arthroplasty. Arch Orthop Trauma Surg 132: 555-563.

14. Kellgren JH, Lawrence JS (1957) Radiological assessment of osteo-arthrosis. Ann Rheum Dis 16: 494-502.

15. Mahoney FI, Barthel DW (1965) Functional Evaluation: The Barthel Index. Md State Med J 14: 61-65.

16. WHO (1977) Manual of the international statistical classification of diseases injuries, and causes of death, Ninth Revision Vol 1.

17. Lopopolo RB, Greco M, Sullivan D, Craik RL, Mangione KK (2006) Effect of therapeutic exercise on gait speed in community-dwelling elderly people: meta-analysis. Phys Ther 86: 520-540.

18. Possiadlo D, Richardson S (1991) The timed "Up \& Go": a test of basic functional mobility for frail elderly persons. J Am Geriatr Soc 39: 142-148.

19. Lord SR, Murray SM, Chapman K, Munro B, Tiedemann A (2002) Sit-to-stand performance depends on sensation, speed, balance, and psychological status in addition to strength in older people. J Gerontol A Biol Sci Med Sci 57: M539543.

20. Katoh M, Yamasaki H (2009) Comparison of reliability of isometric leg muscle strength measurements made using a hand-held dynamometer with and without a restraining belt. J Phys Ther Sci 21: 37-42.

21. Mizner RL, Petterson SC, Snyder-Mackler L (2005) Quadriceps strength and the time course of functional recovery after total knee arthroplasty. J Orthop Sports Phys Ther 35: 424-436.

22. Steffen TM, Hacker TA, Mollinger L (2002) Age- and gender-related test performance in community-dwelling elderly people: Six-Minute Walk Test, Berg Balance Scale, Timed Up \& Go Test, and gait speeds. Phys Ther 82: 128-137.

23. Yeung TS, Wessel J, Stratford PW, MacDermid JC (2008) The timed up and 
Citation: Hiyama Y, Okada S (2014) Risk of Fall in Patients during the Early Months after Total Knee Arthroplasty. J Arthritis 3: 139. doi:10.4172/21677921.1000139

go test for use on an inpatient orthopaedic rehabilitation ward. J Orthop Sports Phys Ther 38: 410-417.

24. MacDougall JD, Wenger HA, Green HJ (1991) Physiological testing of the highperformance athlete. (2nd Edn.), Human Kinetic Publishers, Leeds.

25. Watkins MA, Riddle DL, Lamb RL, Personius WJ (1991) Reliability of goniometric measurements and visual estimates of knee range of motion obtained in a clinical setting. Phys Ther 71: 90-96.

26. Hill KD, Schwarz JA, Kalogeropoulos AJ, Gibson SJ (1996) Fear of falling revisited. Arch Phys Med Rehabil 77: 1025-1029.

27. Delbaere K, Crombez G, van Haastregt JC, Vlaeyen JW (2009) Falls and catastrophic thoughts about falls predict mobility restriction in communitydwelling older people: A structural equation modeling approach. Aging Ment Health 13: 587-592.

28. Tinetti ME, Speechley M, Ginter SF (1988) Risk factors for falls among elderly persons living in the community. N Engl J Med 319: 1701-1707.

29. Nevitt MC, Cummings SR, Kidd S, Black D (1989) Risk factors for recurrent nonsyncopal falls. A prospective study. JAMA 261: 2663-2668.
30. Campbell AJ, Borrie MJ, Spears GF (1989) Risk factors for falls in a communitybased prospective study of people 70 years and older. J Gerontol 44: M112117.

31. Himann JE, Cunningham DA, Rechnitzer PA, Paterson DH (1988) Age-related changes in speed of walking. Med Sci Sports Exerc 20: 161-166.

32. Tinetti ME, Williams TF, Mayewski R (1986) Fall risk index for elderly patients based on number of chronic disabilities. Am J Med 80: 429-434.

33. Fortinsky RH, Panzer V, Wakefield D, Into F (2009) Alignment between balance confidence and fall risk in later life: Has over-confidence been overlooked? Health, Risk \& Society 11: 341-352.

34. Robinovitch SN, Cronin T (1999) Perception of postural limits in elderly nursing home and day care participants. J Gerontol A Biol Sci Med Sci 54: B124-130.

35. Wolfson LI, Whipple R, Amerman P, Kleinberg A (1986) Stressing the postura response. A quantitative method for testing balance. J Am Geriatr Soc 34 845-850. 